\title{
comportement et dimensionnement des colonnes ballastées
}

\author{
par \\ J. Vautrain \\ Laboratoire Régional des Ponts et Chaussées de Rouen
}

\section{Introduction}

Les remblais d'accès au Pont Mathilde qui franchit la Seine en amont de Rouen sont établis sur des sols mous et compressibles. Les problèmes de stabilité et de tassement posés par ces terrains ont conduit à traiter le sol avec des colonnes ballastées dans les zones les plus difficiles et avec des drains de sable ailleurs. Dans un souci d'économie, les culées du pont ont été fondées en tête de chacun de ces remblais. A cette occasion, des appareils de contrôle de tassement de pression interstitielle et de contrainte totale ont été placés à la base des remblais et dans le terrain naturel, afin de suivre le comportement du sol et des colonnes ballastées au cours du temps.

Après un bref historique des études géotechniques qui ont conduit à choisir les colonnes ballastées comme mode de fondation, cet article expose les résultats de ces contrôles. L'interprétation des phénomènes observés qui est ensuite présentée, débouche sur une méthode de calcul permettant de prévoir le comportement de colonnes ballastées.

\section{Les études préalables à la construction}

\subsection{Caractéristiques des ouvrages}

Rive Droite

Le remblai atteint $9,50 \mathrm{~m}$ de hauteur. Sa largeur est de $80 \mathrm{~m}$ en pied de remblai au droit de la culée du pont (fig. 1). A cet endroit, il est interrompu par un mur en terre armée. En arrière du mur, sa largeur croît ensuite rapidement.

\section{Rive Gauche}

La hauteur du remblai atteint $8 \mathrm{~m}$ au droit de la culée du pont et décroît ensuite progressivement jusqu'à s'annuler (fig. 1).

\subsection{Nature et caractéristiques des terrains}

Le long des rives de la Seine, sous une couverture superficielle d'environ $2,50 \mathrm{~m}$ de remblais anciens, s'établit une zone de terrains alluvionnaires dont l'épaisseur atteint 8 à $10 \mathrm{~m}$. Rive Droite de la Seine, le remblai n'est intéressé par ces terrains que sur une longueur de $50 \mathrm{~m}$ (fig. 1). Rive Gauche, la zone est plus étendue, de telle sorte que le remblai se trouve en totalité sur des sols compressibles.

De part et d'autre de la Seine, on distingue, depuis la base des remblais, trois couches principales:

- de la vase ou du limon vaseux sur 1 à $2 \mathrm{~m}$ d'épaisseur;

- de la tourbe très compressible en une couche plus ou moins épaisse, tantôt très polluée par la vase, voire inexistante, tantôt franche et noire, atteignant jusqu'à 2,50 $\mathrm{m}$ d'épaisseur;

- une seconde couche de vase qui s'établit jusqu'aux alluvions sablo-graveleuses sous-jacentes. Sur la Rive Droite, elle peut devenir localement très sableuse, mais reste cependant argileuse jusqu'à la base de la couche. Rive Gauche, le premier mètre est très argileux, puis cette vase devient franchement sableuse, perméable, plus compacte et peu compressible. L'épaisseur d'alluvions compressibles, est donc plus importante sur la Rive Droite $(8$ à $10 \mathrm{~m})$ que sur la Rive Gauche $(6 \mathrm{~m})$.

Ces terrains, à l'origine marécageux, ont été mis hors d'eau avec $2,50 \mathrm{~m}$ de remblais divers. La nappe phréatique est en effet située au voisinage du toit des alluvions fines.

Comme le montre le tableau 1 où sont rassemblées les principales propriétés des sols de fondation, c'est la tourbe qui est la plus compressible. II faut signaler cependant que ces terrains, y compris la tourbe, présentent un rapport $\frac{\sigma_{c}^{\prime}}{\sigma_{v_{0}}^{\prime}}$ voisin de $1.5\left(\sigma_{c}^{\prime}\right.$ pression de préconsolidation, $\sigma_{v_{0}}^{\prime}$ pression verticale des terres) facteur qui contribue à limiter le tassement. 

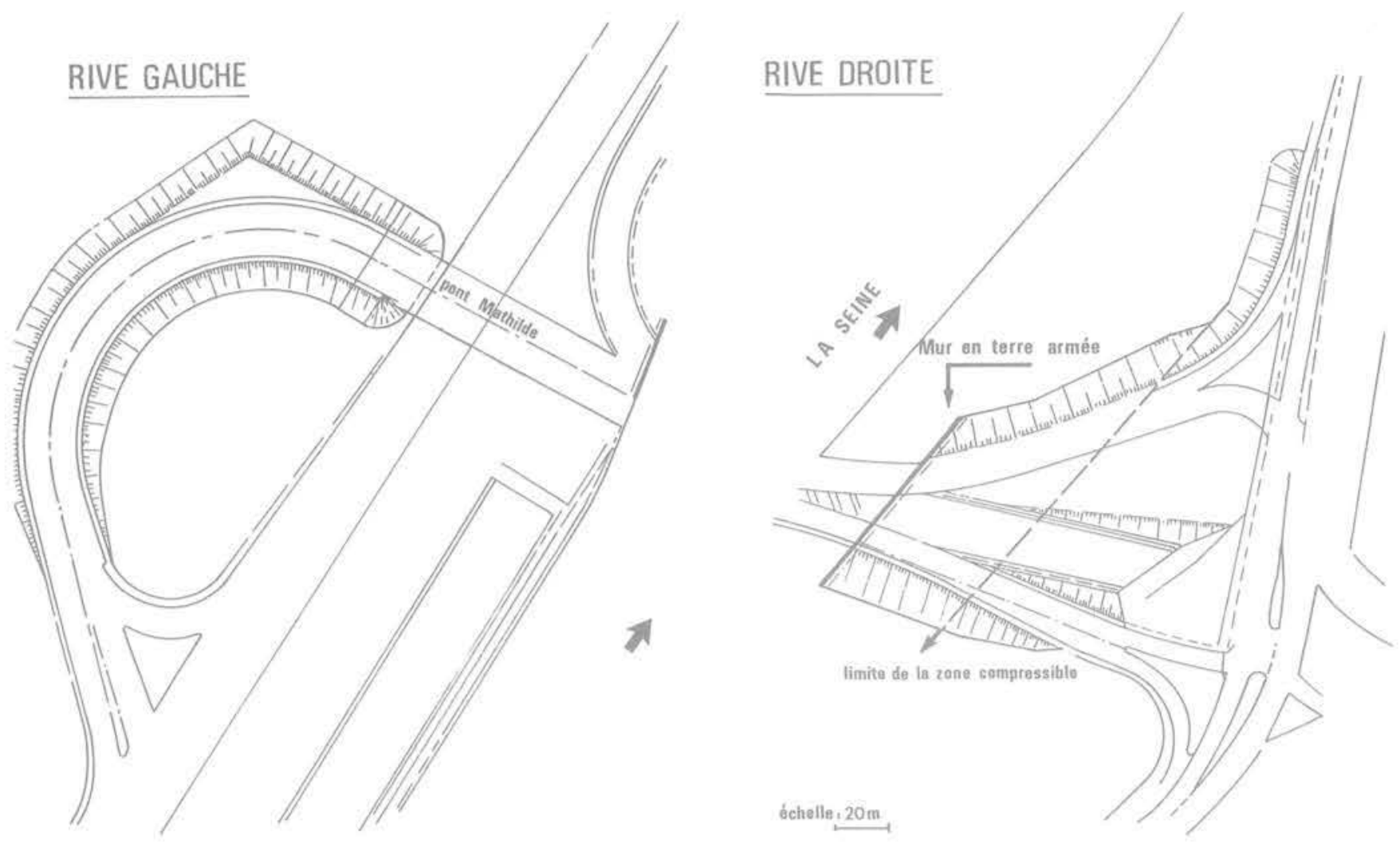

Fig. 1 Plans de situation des remblais

Les cohésions « non drainées »Cu ont été mesurées en laboratoire, à l'essai triaxial, ainsi qu'au scissomètre de chantier. C'est souvent la vase qui donne les résultats les plus faibles et plus particulièrement la couche de vase, non polluée par du sable, située immédiatement au-dessous de la couche de tourbe.

Tableau 1 Principales propriétés des alluvions fines et des remblais superficiels

\begin{tabular}{|c|c|c|c|c|}
\hline $\begin{array}{l}\text { Profondeur } \\
\text { (m) }\end{array}$ & Nature & W \% & $\begin{array}{c}\mathrm{Cu} \\
(\mathrm{kPa})\end{array}$ & $\frac{C_{C}}{1+e_{0}}$ \\
\hline \multirow{2}{*}{$2,50 \mathrm{~m}$} & remblai & 35 à 50 & & \\
\hline & vase & 35 à 50 & $\begin{array}{c}50 \\
a \\
20\end{array}$ & $\begin{array}{c}0,1 \\
\grave{a} \\
0,2\end{array}$ \\
\hline $5,50 \mathrm{~m}$ & tourbe & 150 à 250 & 25 & $\begin{array}{c}0,3 \\
\text { à } \\
0,5\end{array}$ \\
\hline $8,0 \mathrm{~m}$ & vase & 50 & $\begin{array}{c}20 \\
\dot{a} \\
30\end{array}$ & $\begin{array}{c}0,1 \\
\text { à } \\
0,2\end{array}$ \\
\hline $12,0 \mathrm{~m}$ & $\begin{array}{l}\text { sables et } \\
\text { graviers }\end{array}$ & & & \\
\hline
\end{tabular}

1.3 Prévision du comportement du sol sans traitement

Amplitude du tassement

L'amplitude du tassement a été déterminée à l'aide de la relation:

$$
\Delta H=H\left[\frac{C_{s}}{1+e_{0}} \log \frac{\sigma_{c}^{\prime}}{\sigma_{v_{0}}^{\prime}}+\frac{C_{c}}{1+e_{o}} \log \frac{\sigma_{v_{0}}^{\prime}+\Delta \sigma}{\sigma_{c}^{\prime}}\right]
$$

dans laquelle:

$\mathrm{H}$ est l'épaisseur de la couche compressible; $e_{0}$ l'indice des vides avant tassement; $\sigma_{v_{0}}^{\prime}$ la pression verticale des terres; $\sigma_{c}^{\prime}$ la pression de préconsolidation: $\Delta \sigma$ la charge appliquée;

$\mathrm{C}_{\mathrm{s}}$ l'indice de compression entre $\sigma_{\mathrm{v}}^{\prime}$ et $\sigma_{\mathrm{c}}^{\prime}$;

$\mathrm{C}_{\mathrm{c}}$ l'indice de compression pour une charge supérieure à $\sigma_{c}^{\prime}$.

Les résultats des calculs sont présentés dans la figure $n^{\circ} 2$.

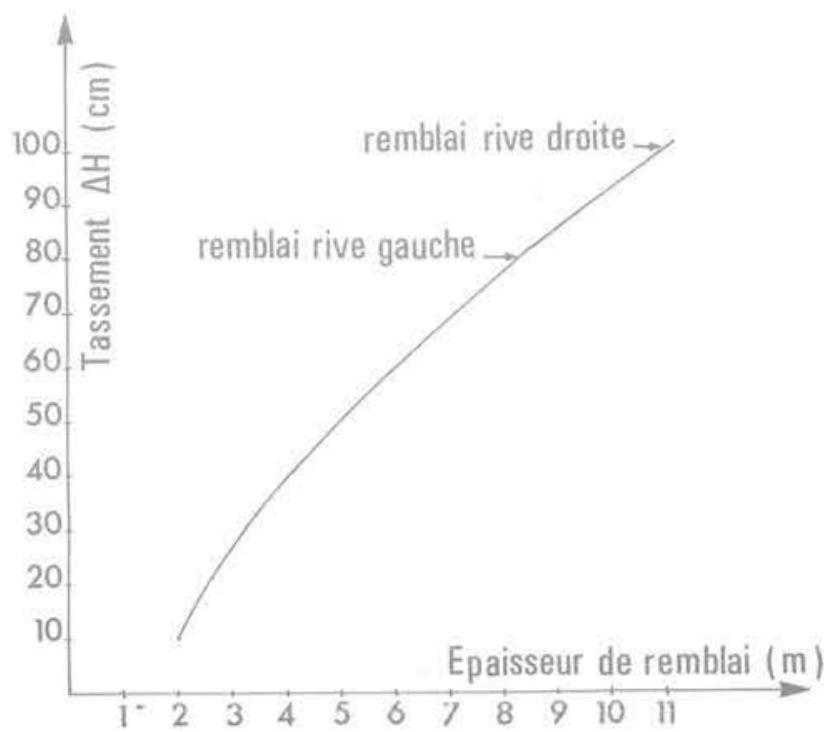

Fig. 2 Tassement en fonction de l'épaisseur de remblai 


\section{Stabilité générale}

Le calcul a été mené à l'aide d'un programme de calcul de stabilité en rupture circulaire (Blondeau - 1976) en simulant une construction des remblais en plusieurs phases. Entre chacune d'elles, il est alors prévu d'atteindre $80 \%$ de la consolidation des sols compressibles.

Les cohésions prises en compte sous les remblais ont donc été majorées au fur et à mesure de leur construction, à l'aide de la relation :

dans laquelle:

$$
\mathrm{Cu}=\mathrm{Cu}_{\mathrm{o}}+\mathrm{U} \cdot \Delta \mathrm{p} \operatorname{tg} \varphi_{\mathrm{cr}}
$$

$\mathrm{Cu}_{0}$ est la cohésion initiale:

$\Delta p$ la surcharge appliquée;

$\cup$ le degré de consolidation (ici $80 \%$ ).

Afin de tenir compte d'éventuelles fissures verticales dans les remblais, la résistance au cisaillement du remblai a été négligée.

Le coefficient de sécurité minimal au-dessous duquel il convient de ne pas descendre, est de 1,5 (Pilot - 1976).

L'ensemble de ces hypothèses conduisait à envisager, sur la rive droite, la construction du remblai en quatre phases, avec en phase finale un coefficient de sécurité voisin de 1, malgré l'amélioration de cohésion due à la consolidation des couches compressibles.

Par contre, rive gauche, la construction pouvait se faire en 3 phases: $4,50 \mathrm{~m}$ de hauteur pour la première, $6,50 \mathrm{~m}$ et $8 \mathrm{~m}$ pour les seconde et troisième phases.

\section{Temps de tassement}

L'ordre de grandeur du temps de tassement a été estimé à l'aide de la relation (Bourges - 1973) :

$$
t=\frac{T_{v} H^{2}}{C_{v}}
$$

où $T_{v}$ facteur temps est égal à 0,57 pour $80 \%$ de consolidation.

$\mathrm{H}$ est le chemin de drainage.

Les valeurs obtenues étant de l'ordre de 300 jours pour un coefficient $\mathrm{C}_{\mathrm{y}}$ moyen de $2,10^{-7} \mathrm{~m}^{2} / \mathrm{s}$, en supposant que les fines couches de sable rencontrées dans les sondages limitaient le chemin de drainage.

\subsection{Conséquences}

Le délai de construction de ces remblais était d'une année. Si l'on envisageait de les construire en plusieurs phases, il était nécessaire d'accélérer la consolidation à l'aide de drains verticaux. Cette solution, satisfaisante pour le remblai Rive Gauche, ne l'était pas pour le mur en terre armée Rive Droite où il restait deux problèmes à résoudre :

- la stabilité ne serait sans doute pas assurée en fin de construction;

- l'amplitude du tassement était vraisemblablement incompatible avec un mur en terre armée. II est conseillé, en effet, de ne pas dépasser un tassement différentiel de $1,5 \%$. Or, entre la crête et le pied du talus, c'est une valeur de $2,5 \%$ qui était attendue.

C'est donc pour résoudre ces deux problèmes qu'il a été envisagé d'améliorer les caractéristiques du sol avec des colonnes ballastées, solution déjà utilisée dans des cas similaires (Jardin - 1974).

\section{Dimensionnement du réseau de colonnes ballastées}

\subsection{Principe des colonnes ballastées}

Un avant-trou est effectué à l'aide d'une aiguille vibrante dont les diamètres habituels varient de $0,25 \mathrm{~m}$ à $0,50 \mathrm{~m}$. Le forage est effectué en vibration, sous le propre poids de l'aiguille, aidé d'une injection d'eau à la base qui permet de réaliser plus rapidement le forage et de le débarasser de tous les éléments fins. Lorsque l'aiguille est arrivée à la profondeur désirée. on introduit le gravier, qui est compacté par vibration au fur et à mesure de la remontée de l'aiguille (fig. 3).

Le compactage est suffisamment intense pour faire pénétrer le matériau dans le terrain. II se forme ainsi une colonne de 0,60 $\mathrm{m}$ à 1,20 $\mathrm{m}$ de diamètre suivant la résistance du sol.

\subsection{Dimensionnement du réseau de colonnes ballastées}

Hypothèses du calcul

Une colonne ballastée est souvent comparée à un pieu. Constituée de matériaux granulaires frottants, elle peut effectivement porter une charge élevée, dans la mesure où le terrain naturel assure un bon frettage. Cependant, à un niveau donné, la charge verticale reprise par la colonne sera fonction de la contrainte horizontale existant dans le terrain naturel. Elle augmentera donc avec la profondeur. Par ailleurs, la pression appliquée par le remblai va se répartir entre le terraiń naturel et les colonnes. Si ces dernières se trouvent chargées de manière excessive, elles vont se déformer et refouler le terrain voisin, ce qui conduit à une augmentation de la contrainte horizontale et par conséquent de la contrainte verticale dans la colonne jusqu'à un nouvel état d'équilibre.

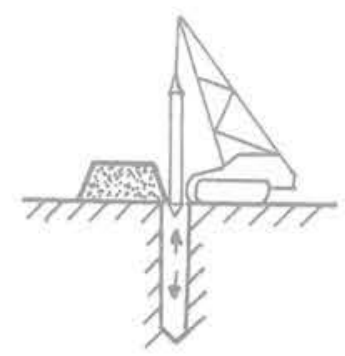

I Enfoncement du vibreur et formation du trou

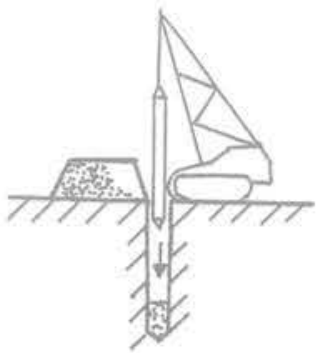

I/ Bourrage en ballast

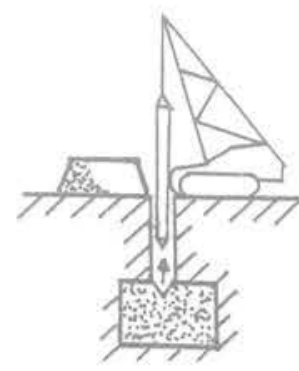

III Compactage et vibration latérale

Fig. 3 
Le comportement d'une colonne ballastée est de ce fait très éloigné de celui d'un pieu. II serait plutôt comparable à celui de l'éprouvette d'un essai triaxial autour de laquelle existerait une contrainte horizontale susceptible de croître avec les déformations. Ainsi, pour une contrainte horizontale donnée $\sigma_{h}^{\prime}$ existe une contrainte verticale $\sigma_{1}^{\prime}$ maximale définie à partir de l'angle de frottement interne $\varphi$ du ballast :

$$
\sigma_{i}^{\prime}=\frac{1+\sin \varphi}{1-\sin \varphi} \sigma_{h}^{\prime}
$$

\section{Dimensionnement}

Pour éviter de grandes déformations, la contrainte $\sigma_{\mathrm{h}}^{\prime}$ a été limitée à la valeur de la pression de fluage mesurée au pressiomètre $100 \mathrm{kPa}$, soit une contrainte verticale $\sigma_{1}^{\prime}$ limite égale à :

$$
\sigma_{1}^{\prime}=580 \mathrm{kPa} \text { avec } \varphi=45^{\circ} \text {. }
$$

Compte tenu du faible espacement des colonnes, la charge appliquée était susceptible d'être reprise en totalité par les colonnes à la manière d'un pieu qui se charge par frottement négatif (Combarieu - 1974).

Enfin, en ce qui concerne la section des colonnes, les entreprises spécialistes de cette technique, consultées à cette occasion, ont estimé qu'il était possible d'obtenir une section de $1 \mathrm{~m}^{2}$ dans ce type de terrain, quitte à accentuer le phénomène de refoulement du sol en augmentant l'injection d'eau.

L'ensemble de ces hypothèses a conduit à répartir les colonnes tous les $3 \mathrm{~m}^{2}$ sur la Rive Droite, soit un espacement de $1,70 \mathrm{~m}$ en maille carrée, avec une contrainte $\sigma_{i}^{\prime}$ de $580 \mathrm{kPa}$. Rive Gauche, où il ne s'agissait que de réduire l'amplitude des tassements résiduels au droit de la culée du Pont, la maille a été choisie arbitrairement à $6 \mathrm{~m}^{2}$,

\section{Vérification de la stabilité générale}

La stabilité générale au glissement circulaire a été vérifiée sur la Rive Droite, en affectant au terrain traité une cohésion «non drainée» fictive $\mathrm{Cu}$, moyenne géométrique des résistances au cisaillement des colonnes et du terrain naturel (fig. 4).

Pour des raisons économiques, le traitement a été limité aux zones périphériques. L'étude a été menée sur ordinateur en faisant donc varier la largeur de la zone traitée. En raison du grand nombre d'hypothèses émises dans le dimensionnement, un coefficient de sécurité de 2 a été choisi. Dans ces conditions, au droit du mur en terre armée, le traitement devait atteindre $18 \mathrm{~m}$ de largeur, y compris un débordement de $3 \mathrm{~m}$ au-delà du parement du mur (fig. 5).

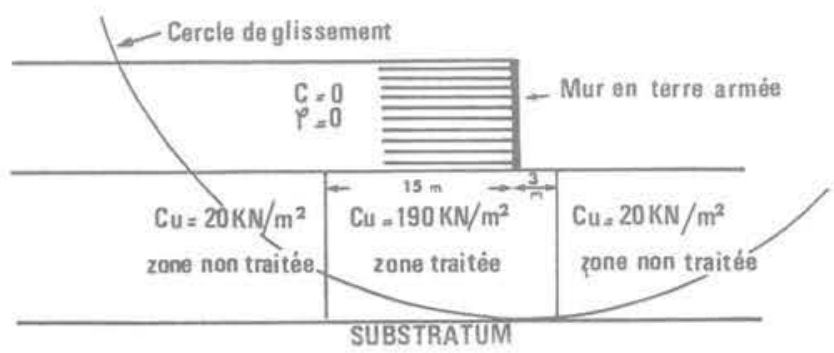

Fig. 4 Etude de la stabilité du mur en terre armée
Compte tenu de la forte cohésion fictive du matériau terre armée, les cercles de rupture passant au niveau des armatures n'ont pas été pris en compte. Enfin, par précaution, la résistance au cisaillement du remblai a été négligée, afin de se prémunir d'éventuelles fissures verticales dues à un déplacement en masse du mur en terre armée vers la Seine.

\section{Réalisation des colonnes ballastées}

\subsection{Le matériel}

La mise en place des colonnes ballastées a été réalisée par l'Entreprise Bachy à l'aide du vibreur électrique torpédo de $350 \mathrm{~mm}$ de diamètre (procédé Keller). Sur la Rive Gauche, les colonnes étaient mises en œuvre une à une, tandis que Rive Droite, deux vibreurs couplés espacés de $1,70 \mathrm{~m}$ permettaient de travailler plus rapidement (fig. 6).

\subsection{Le matériau}

Le matériau utilisé était constitué de $70 \%$ de ballast $(40 / 80)$ et de $30 \%$ d'un tout-venant sableux, de manière à donner à la colonne un caractère drainant.

II faut noter que le sable rend plus difficile l'incorporation des matériaux au terrain et conduit vraisemblablement à une diminution du diamètre de la colonne.

Fig. 6

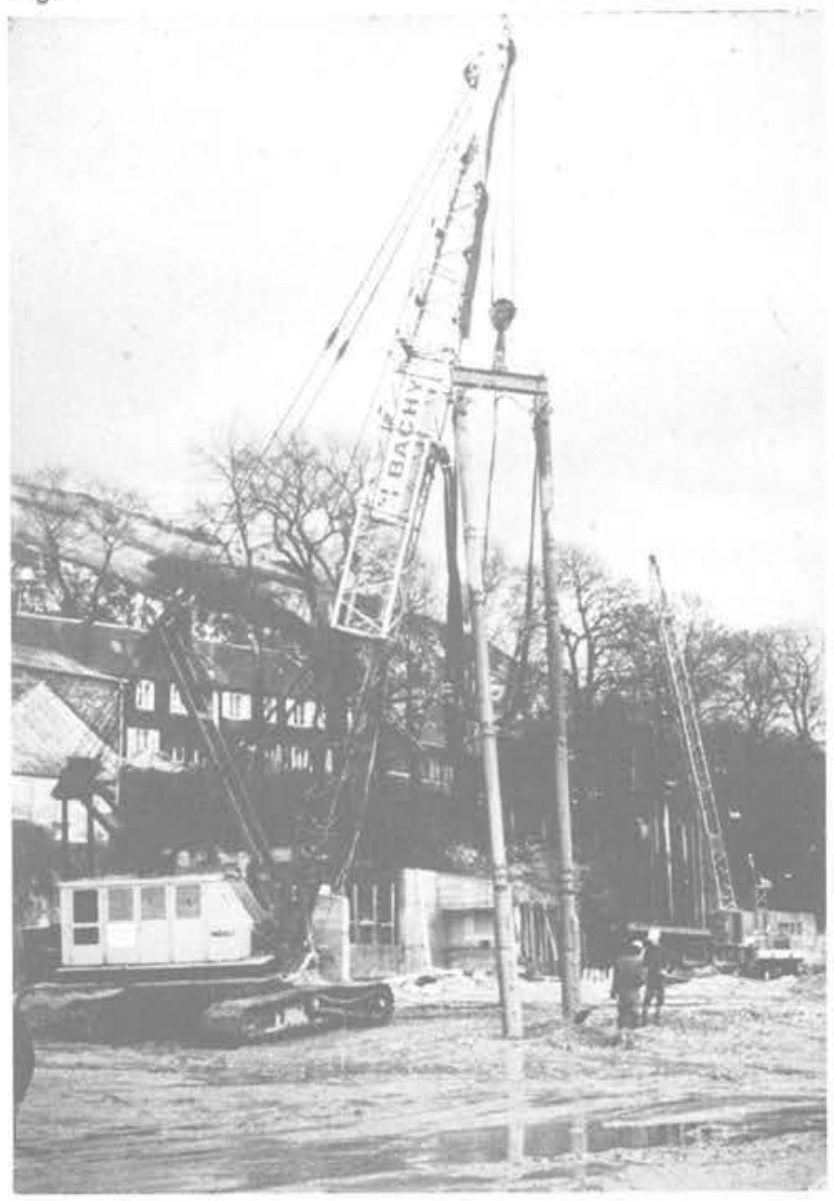




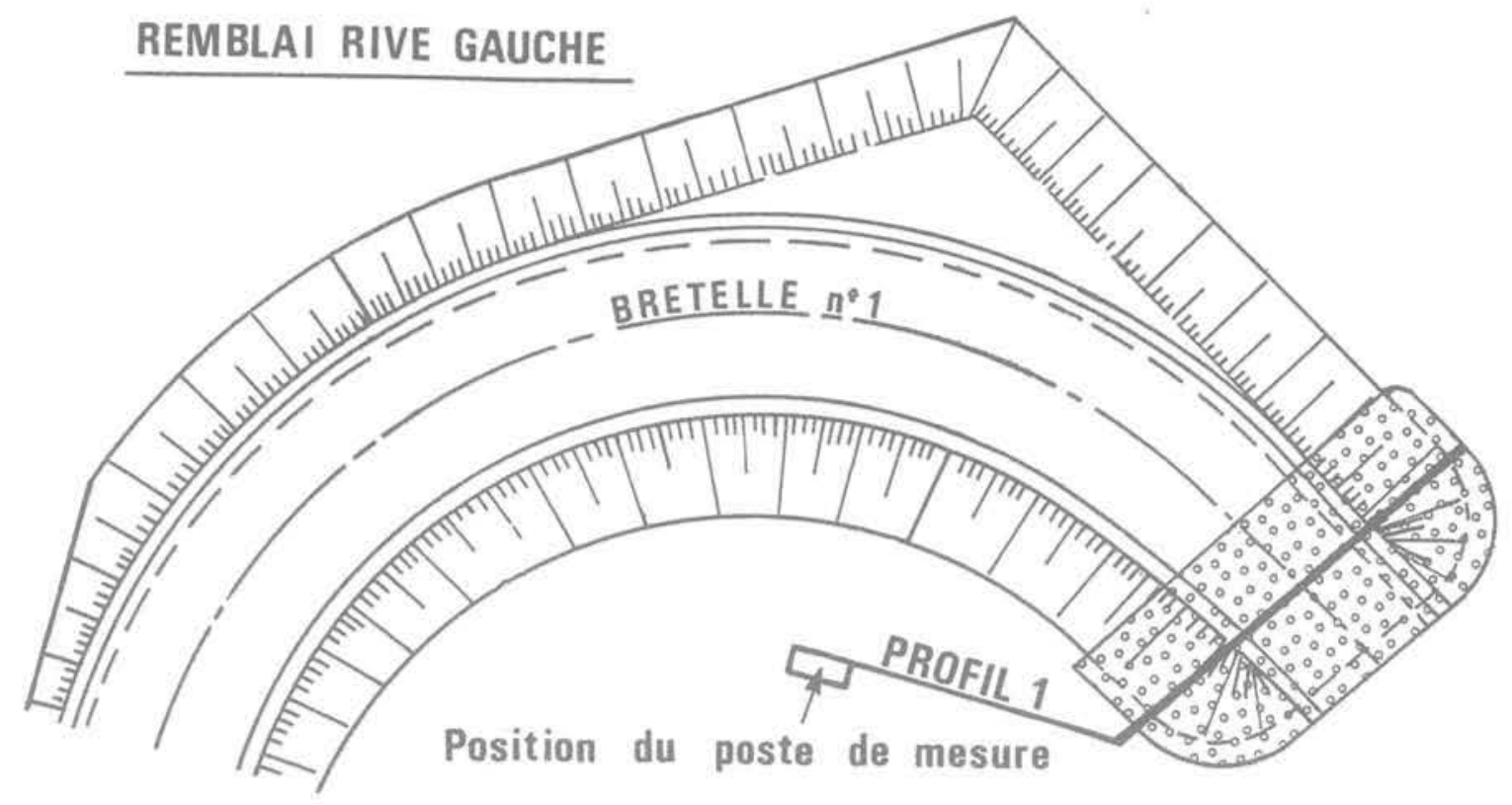

Limite de la zone compressible

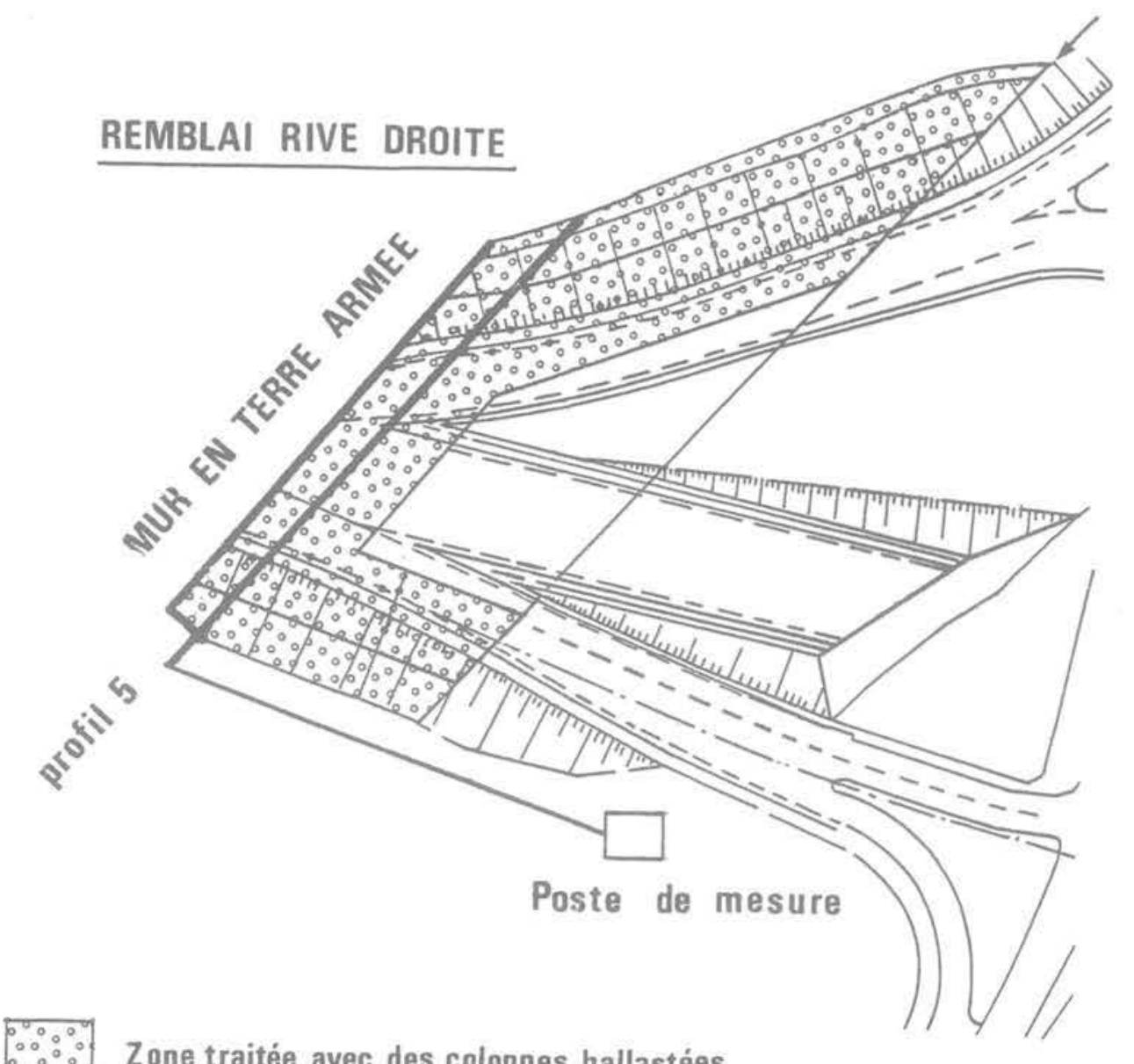

Zone traitée avec des colonnes ballastées 


\subsection{La mise en œuvre}

Le vibreur n'a eu aucune difficulté pour traverser la couche supérieure de remblai malgré la présence de gros éléments, briques, silex, etc.

Le nombre de colonnes réalisées par jour sur la Rive Droite était de 17, soit environ $190 \mathrm{ml}$, tandis que Rive Gauche où un seul vibreur travaillait, le nombre de mètres linéaires était de l'ordre de 70 à 80 .

Le contrôle des quantités de matériaux incorporés au terrain a montré que la section de $1 \mathrm{~m}^{2}$ souhaitée n'était pas atteinte. En moyenne, sur la Rive Droite, 1,25 tonne a été utilisée par mètre linéaire, soit une section moyenne d'environ $0,6 \mathrm{~m}^{2}$.

Cependant, les remblais superficiels et les éboulis de craie ne pouvaient absorber qu'une faible quantité de matériau. II est probable que le diamètre des colonnes était plus important dans les couches les plus molles de vase et de tourbe, mais restait cependant inférieur aux prévisions.

\subsection{Amélioration de la résistance au cisaillement des sols autour des colonnes}

Deux sondages scissométriques ont été réalisés, Rive Droite de la Seine, l'un avant traitement, l'autre après traitement. Les résultats (fig. 7) font apparaître une très forte amélioration de la cohésion. II a même été impossible d'enfoncer les pales du scissomètre au-delà de la couche de tourbe.

\subsection{Stabilité du mur en terre armée}

Compte tenu de la réduction du diamètre des colonnes, la stabilité au glissement circulaire a été vérifiée en tenant compte également de ces essais scissométriques. Les calculs réalisés de la même façon que précédemment ont donné un coefficient de sécurité encore satisfaisant de 1,78.

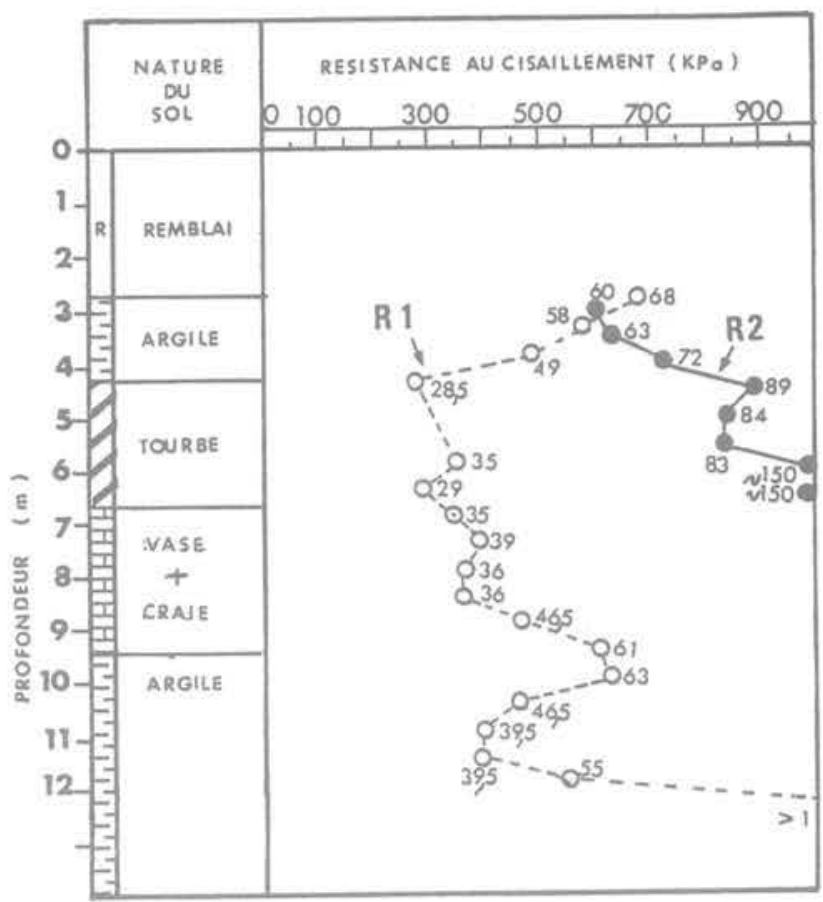

Fig. 7 Sondages scissométriques Rive Droite $R 1$ : avant traitement $R 2$ : après traitement

\section{Dispositifs de contrôle sous les remblais}

Deux profils en travers ont été équipés d'appareils de contrôles (fig. 5).

Le Profil 1, Rive Gauche de la Seine, au droit de la culée du pont.

Le Profil 5, Rive Droite, au niveau du mur en terre armée.

Ces 2 profils sont conformes au schéma des figures $\mathrm{n}^{\circ} 8$ et 9 . On y mesure :

Schémas d'implantation des appareils au profil 5 (profil 1 schéma identique ; remplacer 5 par 1)

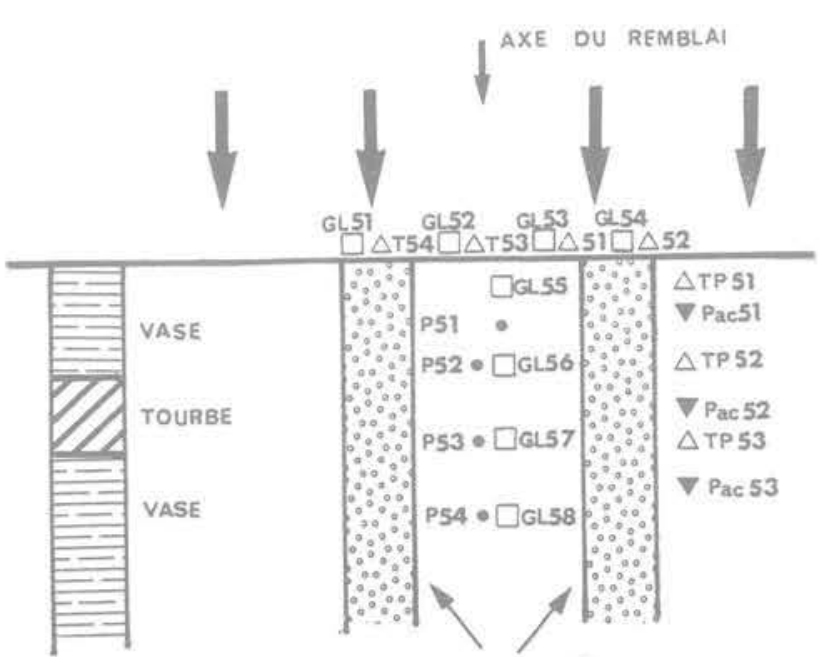

COLONNES BALLASTEES
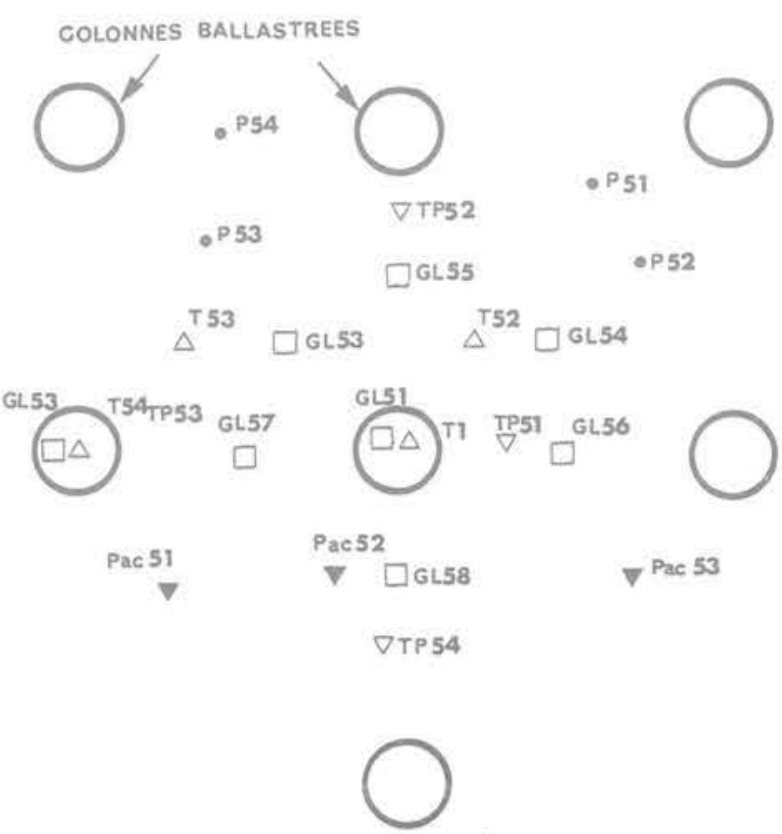

Fig. 9 Position des appareils en plan 
- les contraintes totales verticales à la surface du terrain naturel et les contraintes totales horizontales en profondeur entre les colonnes avec des capteurs Glötzl (GL);

- le tassement en surface et en profondeur (respectivement notés $T$ et TP):

- les pressions interstitielles en profondeur entre les colonnes à l'aide de piézomètres hydrauliques à volume constant $(\mathrm{P})$ et Rive Droite de piézomètres à contre-pression (Pac).

\section{Analyse du comportement des colonnes ballastées-profils 1 et 5}

\subsection{Généralités}

Avant tout chargement, existe dans le terrain naturel et dans les colonnes, un système de contraintes dues au poids des terres et au niveau de la nappe phréatique : $\sigma_{1}^{\prime}=\sigma_{10}^{t}$ : contrainte effective verticale dans la colonne; $\sigma_{v}^{\prime}=\sigma_{v}^{\prime} ;$ contrainte effective verticale dans le terrain naturel;

$\sigma_{h}^{\prime}=\sigma_{h_{0}}^{\prime}$ : contrainte effective horizontale dans le terrain naturel;

$\mathrm{u}=\mathrm{u}_{\mathrm{o}}$ : pression interstitielle due à la nappe phréatique.

La charge verticale $P$ appliquée sur le terrain naturel se répartit entre les colonnes et le terrain naturel. Le système de contraintes devient (fig. 10) :

$$
\begin{aligned}
\sigma_{1}^{\prime} & =\sigma_{i_{0}}^{\prime}+\Delta \sigma_{1}^{\prime} \\
\sigma_{v}^{\prime} & =\sigma_{v_{0}}^{\prime}+\Delta \sigma_{v}^{\prime} \\
\sigma_{\mathrm{h}}^{\prime} & =\sigma_{\mathrm{h}_{0}}^{\prime}+\Delta \sigma_{\mathrm{h}}^{\prime} \\
\mathrm{u} & =\mathrm{u}_{\mathrm{o}}+\Delta \mathrm{u}
\end{aligned}
$$

Dans la colonne qui est constituée d'un matériau perméable, $\Delta u$ reste nul. Par contre, dans le terrain naturel autour de la colonne, l'excès de pression interstitielle $\Delta u$ n'est pas nul. La contrainte effective $\sigma_{h}^{\prime}$ n'a donc pas la même valeur dans la colonne et dans le terrain naturel.

Les capteurs de contrainte mis en place pour contrôler le comportement de colonnes ont mesuré la contrainte totale, à savoir:

$$
\sigma_{1}=\sigma_{\mathrm{s}}^{\prime}+\mathrm{u}
$$

et $\sigma_{\mathrm{y}}=\sigma_{\mathrm{y}}^{\prime}+\mathrm{u}$ à la surface du terrain naturel

$$
\sigma_{h}=\sigma_{h}^{\prime}+u \text {. }
$$

En surface, la pression interstitielle est nulle ou négligeable. En profondeur, il est possible d'obtenir la contrainte effective en déduisant des valeurs de contraintes totales, celles des pressions interstitielles u mesurées.

Au profil 5, les appareils de surface, tassomètres et capteurs Glötzl sont installés au toit des alluvions fines compressibles après décapage des remblais anciens. Par contre, au profil 1 , ces mêmes appareils sont placés au toit de cette couche de remblai qui répartit les contraintes à la manière d'une dalle et masque les phénomènes de surface. C'est par conséquent, le profil 5 qui a servi à étudier plus particulièrement le comportement des colonnes, tandis que le profil 1 permettait de vérifier les interprétations et les hypothèses émises sur le comportement.

\subsection{Analyse des résultats du profil 5}

Dans un souci de clarté, seuls les principaux résultats de tassements de pressions interstitielles et de contraintes totales sont présentés dans la figure 11. II

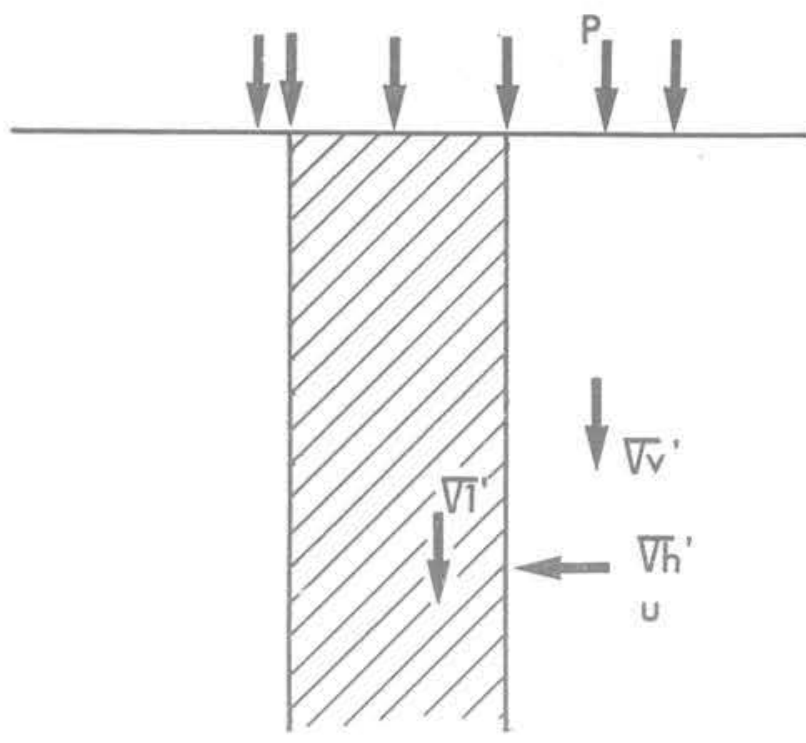

Fig. 10

s'agit donc des accroissements de contraintes totales $\Delta \sigma_{1}^{\prime}$ et $\Delta \sigma_{h}^{\prime}$ et de pressions interstitielles $\Delta u$.

\section{Rythme de chargement}

Le mur en terre armée a été fondé à 1,50 m de profondeur. Les mesures initiales à partir desquelles ont été comptées toutes les variations de pression correspondent donc au terrain naturel déchargé de $1,50 \mathrm{~m}$ de remblai.

A partir du mois d'avril 1976, le mur a été construit sur $8 \mathrm{~m}$ de hauteur, soit $6,50 \mathrm{~m}$ hors terrain naturel. De mai 1976 à juillet 1977, les travaux ont été interrompus. Ils ont repris au mois d'août 1977, et ont amené le mur à $9 \mathrm{~m}$ de hauteur hors sol, soit une épaisseur totale de $10,50 \mathrm{~m}$. II restait à fonder la culée du pont, ainsi qu'à mettre en œuvre les chaussées. Cette dernière phase de travaux est en cours de réalisation.

Résultats des mesures

Tassements

Le tassement à la surface du terrain naturel est identique à celui mesuré en tête des colonnes. Ces dernières se déforment donc de la même amplitude que le sol qui les entoure. La valeur de ce tassement est de l'ordre de $60 \%$ de celle calculée pour un sol non traité.

Au cours de la première phase de chargement, ces déformations se sont stabilisées environ 6 mois après le début du chantier.

Les tassomètres de profondeur ont été placés aux interfaces des principales couches, de manière à pouvoir déterminer le tassement de chacune d'elles. Ils montrent que c'est effectivement la couche de tourbe la plus compressible puisqu'elle provoque plus de $50 \%$ du tassement total, et que ces déformations sont les plus longues à se stabiliser.

\section{Contraintes totales verticales en surface}

Les capteurs placés en tête des colonnes donnent des valeurs 2 à 4 fois plus élevées que ceux installés sur le terrain naturel. II y a donc à la base du remblai des voûtes ae contraintes caractéristiques d'un report de charges sur les colonnes. 


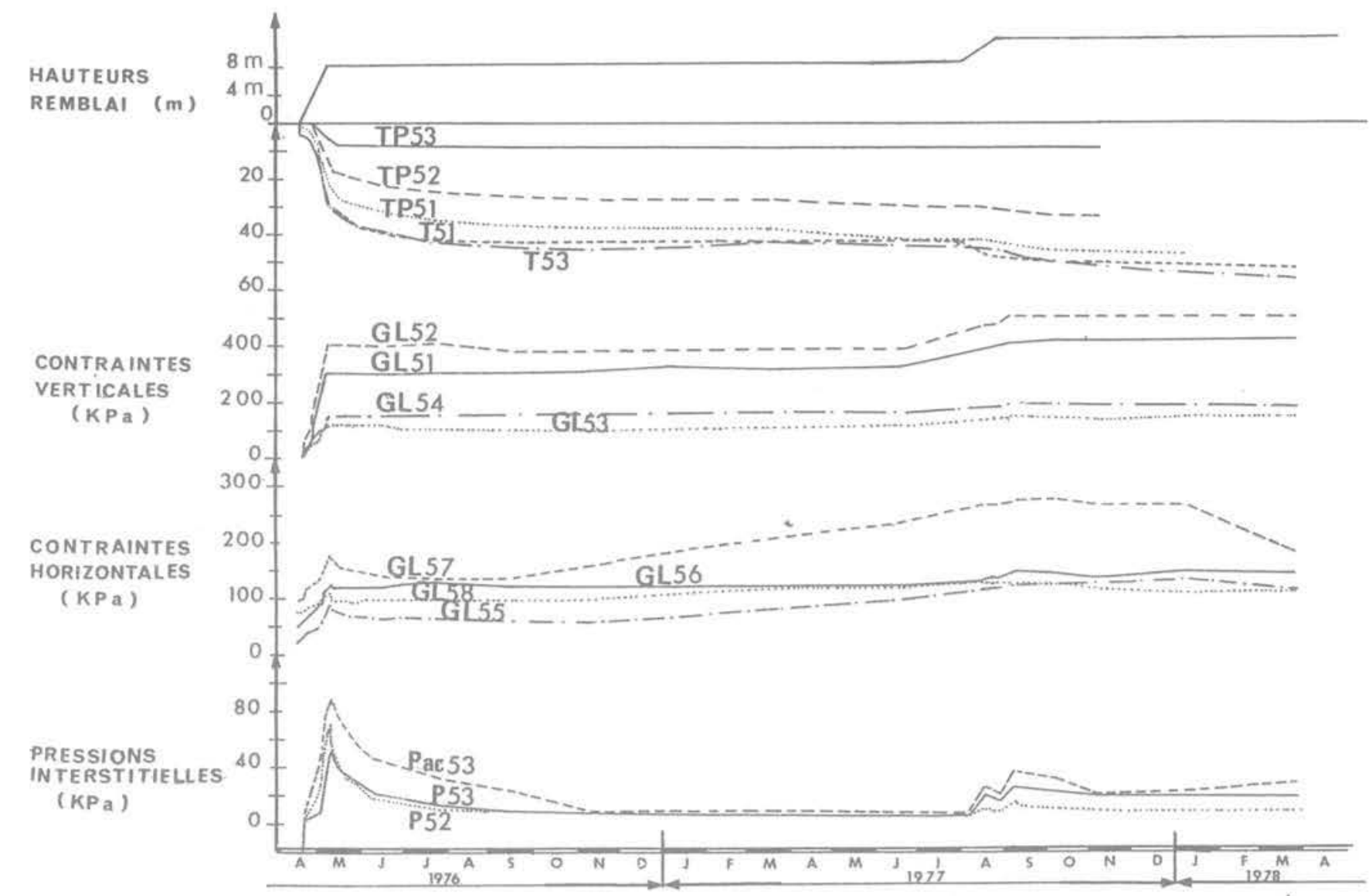

Fig. 11 Résultats des mesures profil 5

Fig. 12

HAUTEUR

DE REMBLAI (m)

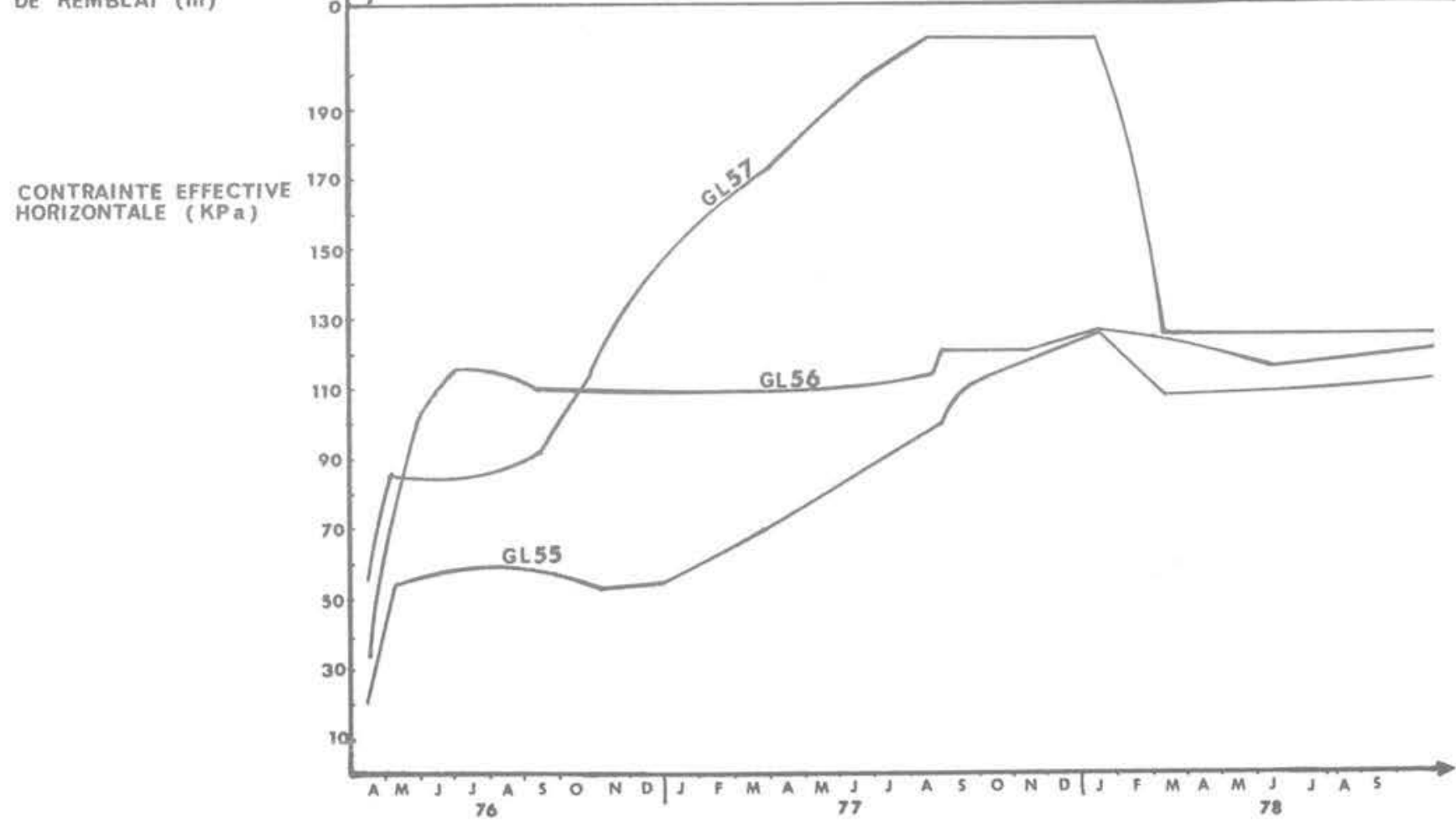


Contraintes horizontales

et pressions interstitielles en profondeur

Les contraintes effectives horizontales déduites des mesures de contraintes totales et de pressions interstitielles sont présentées dans la figure $n^{\circ} 12$. Le capteur GL 56 fait apparaître une augmentation de $\sigma_{h}^{\prime}$ pendant la dissipation des pressions interstitielles, puis une stabilisation apparemment définitive. Par contre, les capteurs GL 55 et surtout,GL 57 , montrent qu'après une rapide évolution de la contrainte au cours du chargement, celle-ci reste'stable pendant toute la phase de dissipation des pressions interstitielles, puis augmentent à nouveau. En ce qui concerne le capteur GL 57, il pourrait s'agir d'un mauvais fonctionnement; cependant, au cours du second chargement, cet appareil est resté très sensible aux variations de contraintes et indique à la fin de cette période, une chute brutale de la contrainte jusqu'à une valeur voisine de celles fournies par les autres capteurs.

Pendant cette phase d'accroissement de contrainte effective horizontale, les pressions interstitielles sont stables et les tassements n'évoluent quasiment pas.

En 1978, quelques mois après le second chargement, les contraintes effectives horizontales sont toutes du même ordre de grandeur.

\section{Interprétation des résultats Tassement}

Aucune prévision correcte du tassement n'avait été établie. Seule une estimation grossière basée sur un module de $30000 \mathrm{kPa}$ mesurée sur la courbe effortdéformation d'un essai triaxial effectué sur la fraction sableuse du matériau, conduisait une valeur de $10 \mathrm{~cm}$ en première phase (Vautrain - 1977).

Le tassement obtenu dépasse largement cette estimation et oblige à examiner le fonctionnement des colonnes ballastées sous l'angle d'un comportement plastique. Dans ce cas, le déviateur de rupture aurait été atteint en tout ou partie de chaque colonne.

Deux facteurs permettent de s'en assurer :

- la contrainte effective horizontale $\sigma_{h}^{\prime}$ mesurée dans la tourbe par exemple est égale à $85 \mathrm{kPa}$ pendant les quelques mois qui suivent le premier chargement. Cette valeur conduit à une contrainte verticale $\sigma_{1}^{\prime}$ limite dans la colonne de $493 \mathrm{kPa}$ pour un angle $\varphi$ de $45^{\circ}$;

- les colonnes sont très rapprochées et s'il s'agissait de pieux en béton, la totalité de la charge appliquée serait reprise par les colonnes par frottement négatif, y compris le poids des terres (Combarieu - 1974). Au niveau de la tourbe, la contrainte verticale induite dans la colonne serait très supérieure à $500 \mathrm{kPa}$, même avec une section de $1 \mathrm{~m}^{2}$. II faut donc admettre que les déformations du terrain naturel autour des colonnes ont amené le déviateur $\sigma_{1}^{\prime}-\sigma_{h}^{\prime}$ à sa valeur limite à partir de laquelle elles sont entrées en plasticité et ont suivi les déformations du terrain naturel sans opposer de résistance.

II est par conséquent normal de mesurer des tassements aussi élevés. Ils restent cependant inférieurs à ceux que l'on obtiendrait sans traitement, parce que la colonne prend une fraction importante de la charge appliquée et décharge en partie le terrain naturel.

Cette analyse valable dans la tourbe, l'est également au niveau du capteur GL 55. Par contre, le capteur GL 56 indique une valeur $\sigma_{h}^{\prime}$ beaucoup plus élevé de $110 \mathrm{kPa}$.
Cependant, la section de $1 \mathrm{~m}^{2}$ n'étant pas atteinte, il est quasiment certain que la colonne s'est trouvée également en plasticité à ce niveau.

\section{Comportement à court terme et à long terme}

Les surpressions interstitielles qui apparaissent dans le sol au cours du chargement se dissipent lentement. Tant qu'elles existent, elles accroissent, le frettage de la colonne et lui permettent de porter à court terme une charge plus élevée que celle déterminée à partir de la contrainte effective horizontale. Lorsque la surpression interstitielle $\Delta u$ se dissipe, elle ne se trouve pas compensée par une augmentation équivalente de la contrainte effective. La force portante à court terme de la colonne est alors décroissante.

Lorsque l'excès de pression interstitielle $\Delta u$ s'est dissipé, la force portante à long terme est atteinte. Les tassements se sont alors stabilisés. Cependant, les contrôles signalent de fortes variations de contraintes effectives horizontales dues vraisemblablement à la mise en butée du sol autour des colonnes. Ce phénomène accroît la portance des colonnes et conduit à une meilleure stabilisation du tassement.

Après le second chargement, une partie de la butée, semble-t-il, disparait, mais il subsiste en particulier pour GL55 une augmentation importante de la contrainte horizontale favorable à la stabilité de l'ensemble.

\subsection{Analyse des résultats du profil 1}

Les principaux résultats sont présentés dans la figure $n^{\circ} 13$ de la même façon qu'au profil 5 .

Sur la Rive Gauche, les colonnes sont réparties à raison de une pour $6 \mathrm{~m}^{2}$ en maille carrée, soit un réseau de $2,5 \mathrm{~m}$ de côté.

Les résultats obtenus présentent certaines analogies avec ceux de la Rive Droite avec cependant deux particularités :

- les mesures de contraintes et de tassements en tête des colonnes sont réalisées au toit des remblais anciens dont l'épaisseur atteint 2,50 à $3 \mathrm{~m}$. II s'en suit une répartition quasi uniforme des contraintes verticales de surface dont l'amplitude est voisine de la charge appliquée, à l'exception d'un capteur dont les résultats nettement plus faibles, laissent penser à un fonctionnement défecteux;

- les mesures de pressions interstitielles mettent en évidence une communication de la nappe des alluvions fines avec celles de la grave sous-jacente. On mesure, en effet, les fluctuations périodiques de la nappe des alluvions fines conformes à celles de la grave et de la Seine, cette dernière étant soumise au marnage. Entre les colonnes, il n'apparaît donc pas de surpressions interstitielles liées au chargement, soit parce que les capteurs sont situés à proximité immédiate des colonnes, soit parce qu'il existe des couches sableuses drainantes dans les alluvions fines imperméables.

Les contraintes totales horizontales mesurées en profondeur évoluent au début de la même manière que les pressions interstitielles. Elles fluctuent autour d'une valeur moyenne croissante. Après le chargement, les contraintes restent constantes pendant un à 2 mois, puis augmentent de façon importante, rapidement pour GL 16 et GL 17, plus lentement mais de même amplitude pour GL 18. Cette évolution se produit pour des déformations quasiment nulles. 


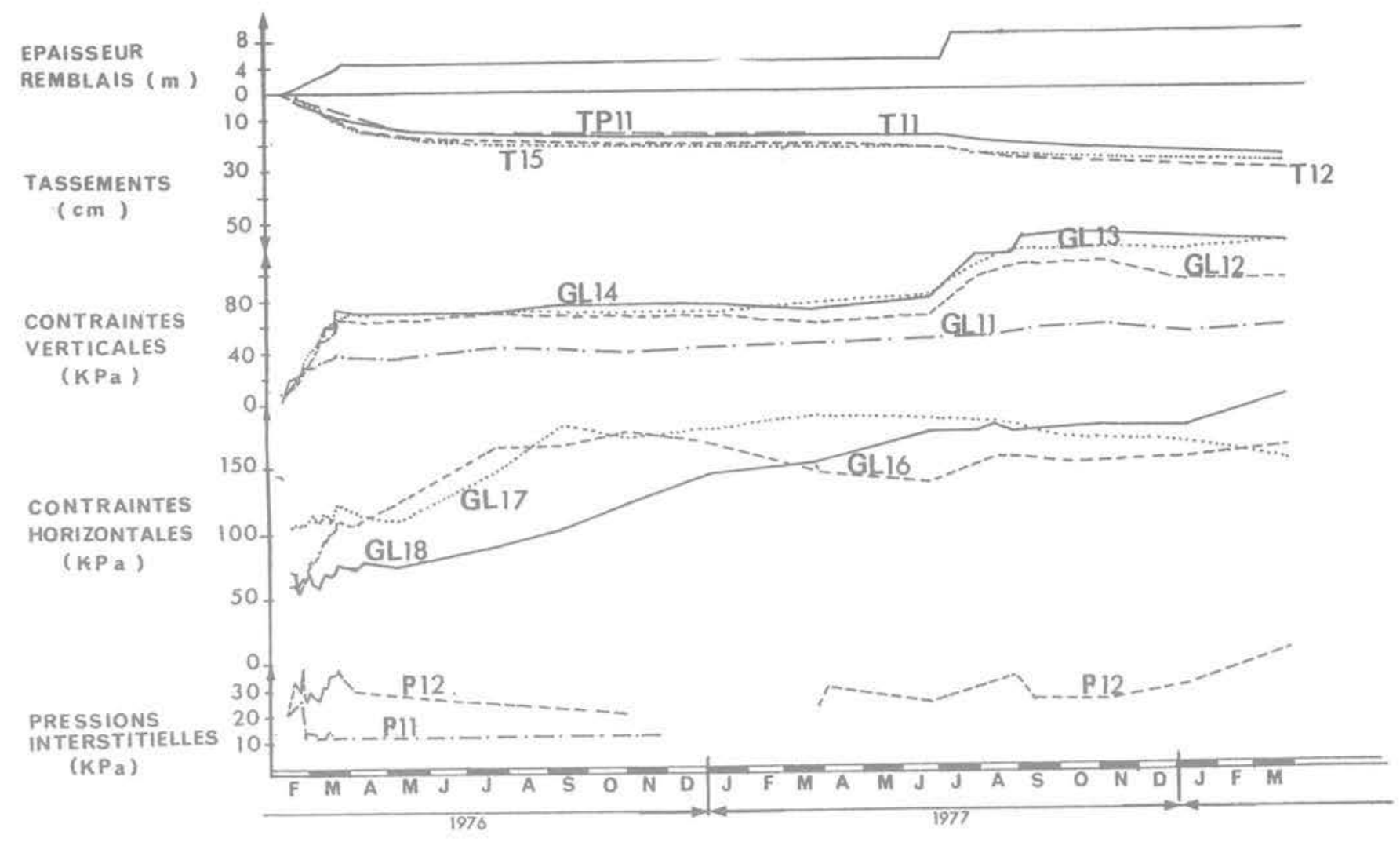

Fig. 13 Résultats des mesures profil 1

De la même manière que Rive Droite, on pourrait vérifier qu'à la fin du premier remblaiement, les colonnes sont incapables de reprendre la totalité de la charge appliquée. Elles sont donc soumises à des déformations plastiques qui s'arrêtent lorsque les terrains voisins se sont consolidés. On assiste alors à une redistribution des contraintes qui conduit à une augmentation considérable de $\sigma_{h}^{\prime}$ permettant à la colonne de supporter une nouvelle charge sans déformations excessives, comme le montrent les résultats obtenus au cours du second chargement. C'est ici l'ensemble des couches compressibles qui sont intéressées par ce phénomène comparable à celui constaté dans la tourbe Rive Droite. Les observations réalisées sur la Rive Gauche semblent donc confirmer l'analyse des résultats de la Rive Droite. D'une manière générale, on peut en conclure :

- que les colonnes ont été soumises à des déformations plastiques au cours de la première étape de chargement. Ces déformations ne s'arrêtent qu'après la consolidation du terrain naturel, c'est-à-dire qu'elles se produisent pendant la période de dissipation des pressions interstitielles;

- qu'il se produit ensuite une mise en butée du terrain caractérisée par l'augmentation des contraintes effectives horizontales et conduisant à un accroissement de la force portante des colonnes;

- que l'étude du comportement à long terme des colonnes ballastées doit être faite à partir de l'analyse de l'évolution des contraintes effectives et non à l'aide des contraintes totales comme cela avait été fait au cours des études préalables. En particulier, il semble donc que pour prevoir le comportement des colonnes, I'utilisation des résultats des essais pressiométriques ne soit pas totalement satisfaisante (Vautrain - 1977).

\section{Conséquences - prévisions du comportement de colonnes ballastées}

\subsection{Analogie avec l'éprouvette triaxiale}

La courbe effort-déformation d'une éprouvette soumise à un essai d'écrasement triaxial présente schématiquement trois parties (fig. 14).

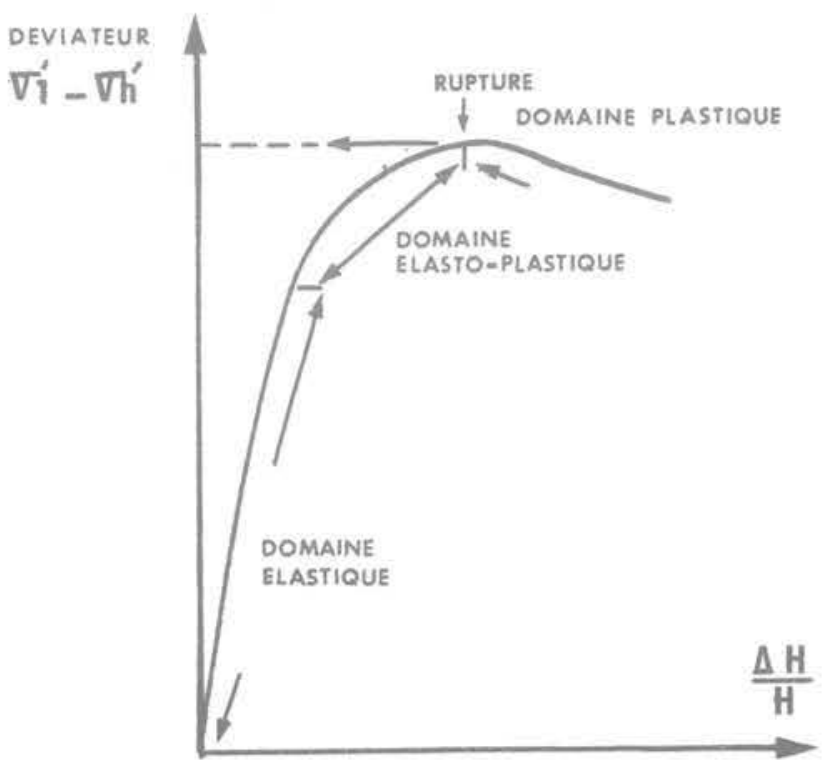

Fig. 14 Courbe effort-déformation d'une éprouvette triaxiale 
Dans la première partie de la courbe, les déformations sont élastiques, c'est-à-dire définies à l'aide du module D'Young $E$.

La seconde partie, juste avant la rupture, est le siège de déformations élastoplastiques.

Dans la troisième partie au-delà de la rupture, il s'agit du domaine des déformations plastiques.

Par analogie avec l'éprouvette triaxiale, une colonne ballastée peut également se comporter de ces trois façons selon la valeur du déviateur à laquelle elle se trouve soumise.

\subsection{Principe du calcul prévisionnel}

- Dans le domaine élastique, la colonne se déforme conformément à son module E. Quel que soit le tassement du terrain naturel, ce sont les contraintes effectives horizontales $\sigma_{i}^{\prime}$ et verticales $\sigma_{h}^{\prime}$ qui déterminent l'amplitude du tassement.

- Dans le domaine élastoplastique, le principe du calcul est identique à celui énoncé ci-dessus, à condition de connaître le module de déformation moyen de la colonne pour le déviateur $\sigma_{1}^{\prime}-\sigma_{h}^{\prime}$ à laquelle elle sera soumise.

- Si le déviateur de rupture est atteint, la colonne est le siège de déformations plastiques. Dans ce cas, la colonne se déforme de la même amplitude que le terrain naturel. II suffit donc de calculer les déformations du sol pour déterminer celles de la colonne.

La valeur du tassement sera par conséquent fonction de l'accroissement de contrainte verticale $\Delta \sigma_{\mathrm{v}}^{\prime}$ apparaissant dans le terrain à la suite du chargement après dissipation des pressions interstitielles. II est possible de le calculer à l'aide des caractéristiques de compressibilité mesurées à l'œdomètre et évidemment de la contrainte effective verticale $\sigma_{v}^{\prime}$.

\subsection{Méthodes de calcul}

\section{Calcul des contraintes}

Dans tous les cas, il est nécessaire de prévoir les contraintes verticales $\sigma_{1}^{\prime}$ et horizontales $\sigma_{h}^{\prime}$, afin de savoir dans quel domaine de déformabilité se situera la colonne.

La valeur de $\sigma_{h}^{\prime}$ à prendre en compte doit être égale à celle qui existe autour de la colonne avant le chargement, après la réalisation des colonnes.

La valeur de $\sigma_{1}^{\prime}$ peut être estimée en supposant que toute la charge est reprise par frottements négatifs par les colonnes, y compris le terrain qui surmonte le niveau considéré. II faut donc connaître le poids spécifique de chacun des matériaux et la section des colonnes.

Cependant, il n'existe pas jusqu'à présent d'éléments permettant de prévoir le diamètre des colonnes. II faut donc s'en référer à l'expérience et vérifier sur le chantier que les quantités de matériaux incorporés dans le sol sont bien conformes aux prévisions.

La valeur de $\sigma_{1}^{\prime}$ ainsi obtenue sera comparée à celle qui conduit au déviateur de rupture. Pratiquement, il est difficile de déterminer cette contrainte limite à partir d'un essai de compression triaxial, compte-tenu de la granulométrie du matériau. II faut donc l'estimer à l'aide de l'angle de frottement interne $\varphi$ du matériau.
Calcul des déformations

Cas des comportements élastique et élastoplastique

Dans ces deux cas, il sera nécessaire de connaître le module de la colonne pour le déviateur $\sigma_{1}^{\prime}-\sigma_{b}^{\prime}$ correspondant. Cependant, comme il est difficile de réaliser un essai de compression triaxial, le moyen le plus sûr consisterait à effectuer un essai de chargement en vraie grandeur et de calculer le module pour les contraintes que les colonnes supporteront réellement.

Dans cet essai, il serait inutile de dépasser la valeur limite de la contrainte $\sigma_{1}^{\prime}$ déterminée ci-dessus, puisqu'au-delà de cette contrainte, les phénomènes de consolidation dans le terrain naturel deviendraient prépondérants.

Le tassement des colonnes est alors prévu en utilisant la loi de Hooke:

$$
\frac{\Delta \mathrm{H}}{\mathrm{H}}=\frac{1}{\mathrm{E}}\left(\sigma_{1}^{\prime}-2 v \sigma_{\mathrm{h}}^{\prime}\right)
$$

avec $\mathrm{E}$ module de déformation du ballast $v$ coefficient de Poisson.

\section{Cas d'un comportement plastique}

Le tassement dû à la consolidation du sol nature devient prépondérant.

L'amplitude est alors calculée à l'aide des relations :

$$
\frac{\Delta H}{H}=H\left[\frac{V_{s}}{1+e_{0}} \log \frac{\sigma_{c}^{\prime}}{\sigma_{v_{0}}^{\prime}}+\frac{C_{c}}{1+e_{0}} \log \frac{\sigma_{v_{0}}^{\prime}+\Delta \sigma_{v}^{\prime}}{\sigma_{c}^{\prime}}\right]
$$

lorsque $\sigma_{v_{0}}^{\prime}+\Delta \sigma_{v}^{\prime}$ est supérieur à $\sigma_{c}^{\prime}$ (cf. $\left.\S 1.3 .1\right)$.

et $\quad \frac{\Delta H}{H}=H \frac{C_{s}}{1+e_{0}} \log \frac{\sigma_{v_{0}}^{\prime}+\Delta \sigma_{v}^{\prime}}{\sigma_{v_{0}}^{\prime}}$

si $\sigma_{v_{0}}^{\prime}+\Delta \sigma_{v}^{\prime}$ est inférieur à $\sigma_{c}^{\prime}$

La répartition des contraintes entre les colonnes et le terrain naturel conduit à l'égalité suivante :

$$
\Delta \sigma_{v}^{\prime}(\mathrm{S}-\mathrm{s})+\mathrm{s} \sigma_{1}^{\prime}=\mathrm{SP}
$$

dans laquelle $S$ est la maille du réseau s la section des colonnes $\mathrm{P}$ la contrainte appliquée en surface

soit

$$
\Delta \sigma_{v}^{\prime}=\frac{S P-S \sigma_{1}^{\prime}}{S-S}=f\left(\sigma_{1}^{\prime}\right) .
$$

Dans cette relation, $\sigma_{1}^{\prime}$ est la contrainte verticale dans la colonne. Comme elle se trouve en plasticité, elle est voisine de la contrainte de rupture définie par:

$$
\sigma_{1}^{\prime}=\frac{1+\sin \varphi}{1-\sin \varphi} \sigma_{h}^{\prime}
$$

$\Delta \sigma_{v}^{\prime}$ est donc une fonction de $\sigma_{n}^{\prime}$, dont la valeur devrait être égale à celle existant dans le terrain autour des colonnes avant le chargement.

\section{Vérifications et applications}

\subsection{Remblai Rive Droite}

Contrainte horizontale $\sigma_{h}^{\prime}$

Dans la figure $n^{\circ} 15$, sont tracées les courbes de tassement relatif $\frac{\Delta H}{H}$ en fonction de la contrainte 


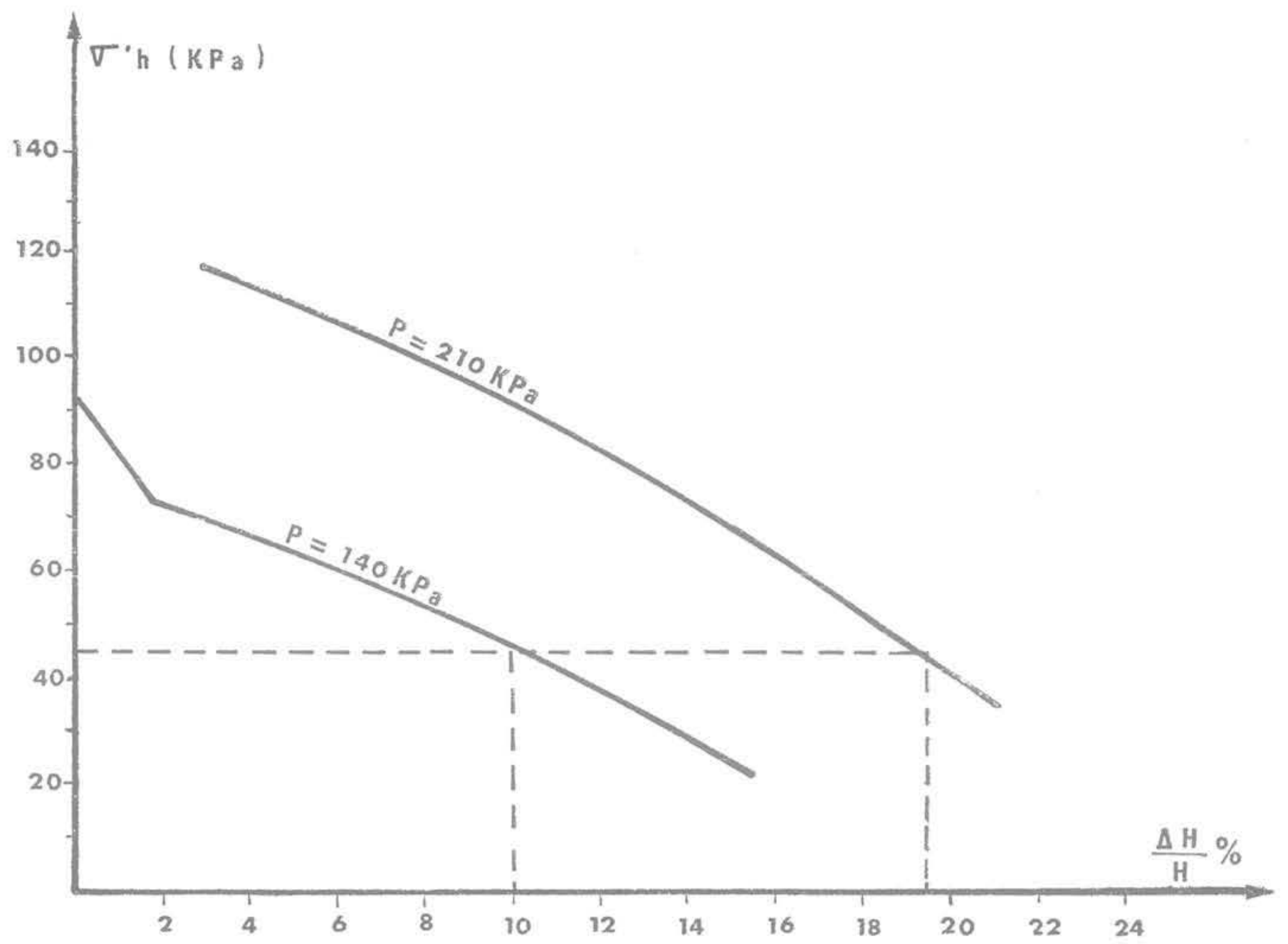

Fig. 15 Tassements relatifs en fonction de $\sigma$ 'h. Couche de tourbe Rive Droite

Fig. 16

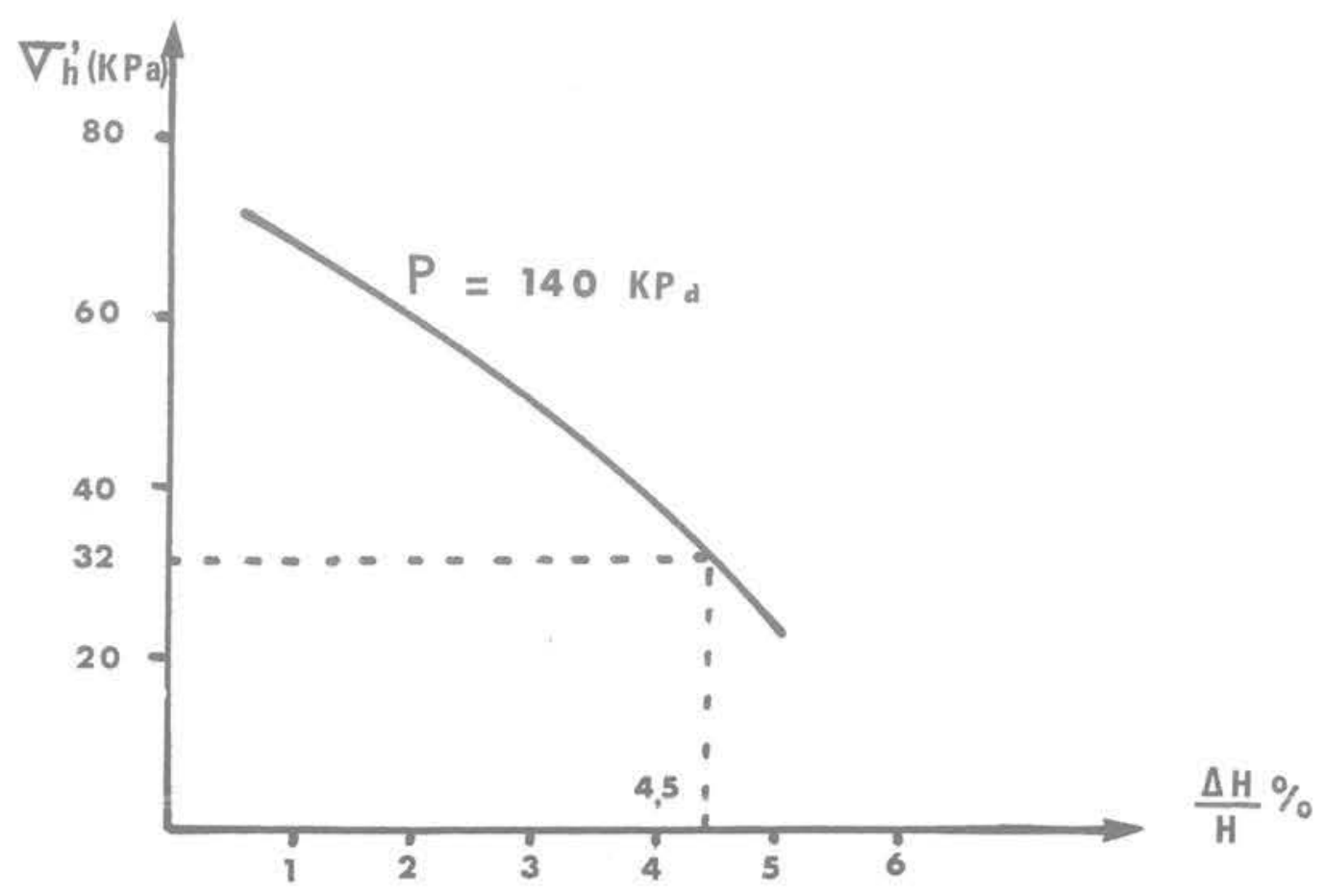


effective $\sigma_{h}^{\prime}$, pour les valeurs de 140 et $210 \mathrm{kPa}$ relatives aux première et seconde phases de consolidation. Les caractéristiques de compressibilité prises en compte sont celles de la tourbe :

$$
\begin{aligned}
& \frac{C_{1}}{1+e_{o}}=0,1 \quad \sigma_{v_{0}}=80 \mathrm{kPa} \\
& \frac{C_{c}}{1+e_{0}}=0,5 \quad \sigma_{c}^{\prime}=120 \mathrm{kPa} .
\end{aligned}
$$

A la verticale du tassement relatif de $10 \%$, valeur obtenue dans la couche de tourbe à la fin du premier chargement, le point représentatif situé sur la courbe $\mathrm{P}=140 \mathrm{kPa}$, correspond à une contrainte horizontale $\sigma_{\mathrm{h}}^{\prime}$ de $48 \mathrm{kPa}$ voisine de celle effectivement mesurée dans le sol avant la construction du remblai (cf. fig. 11, GL. 57).

De la même façon, dans la figure $n^{\circ} 16$, a été tracée la courbe de tassement relatif $\frac{\Delta H}{H}$ en fonction de $\sigma_{h}^{\prime}$ de la couche de vase surmontant la tourbe dans le cas de chargement correspondant à la première étape de construction $(140 \mathrm{kPa})$. Cans cette couche, a été mesurée une contrainte horizontale $\sigma_{\mathrm{h}}^{\prime}=32 \mathrm{kPa}$ (capteur GL 56). Pour cette contrainte, le tassement relatif est de $4,5 \%$, soit une valeur également voisine de celui qui a été mesuré sur le chantier.

\section{Tassement total}

La figure $n^{\circ} 13$ permet aussi de constater que si la construction du remblai s'était effectué en une seule étape, le tassement de la courbe aurait été de l'ordre de 18 à $20 \%$. II semble que le fait d'avoir construit le remblai en 2 phases ait réduit le tassement total.

Au tassement de consolidation qui peut être déterminé de cette façon, il faut ajouter le tassement élastique qui intervient avant d'atteindre l'état limite de la colonne. Ce tassement est faible. Dans le cas de la tourbe par exemple avec un module de $30000 \mathrm{kPa}$, il serait inférieur à $0,7 \%$.

\section{Efficacité du traitement}

L'efficacité du traitement peut être mise en évidence avec la courbes 1 et 2 de la figure $n^{\circ} 17$. La courbe 1 donne le tassement relatif de la couche de tourbe sans traitement et la seconde, le tassement relatif $\frac{\Delta H}{H}$ avec traitement. Ces courbes montrent que c'est à partir de $60 \mathrm{kPa}$ de contrainte $P$ en surface que la colonne rentre en plasticité. A partir de $100 \mathrm{kPa}$, la pression de préconsolidation $\sigma_{c}^{\prime}$ est dépassée et le tassement croît rapidement. L'efficacité du traitement diminue alors pour être nulle aux environs de $P=250 \mathrm{kPa}$.

Fig. 17 Efficacité du traitement

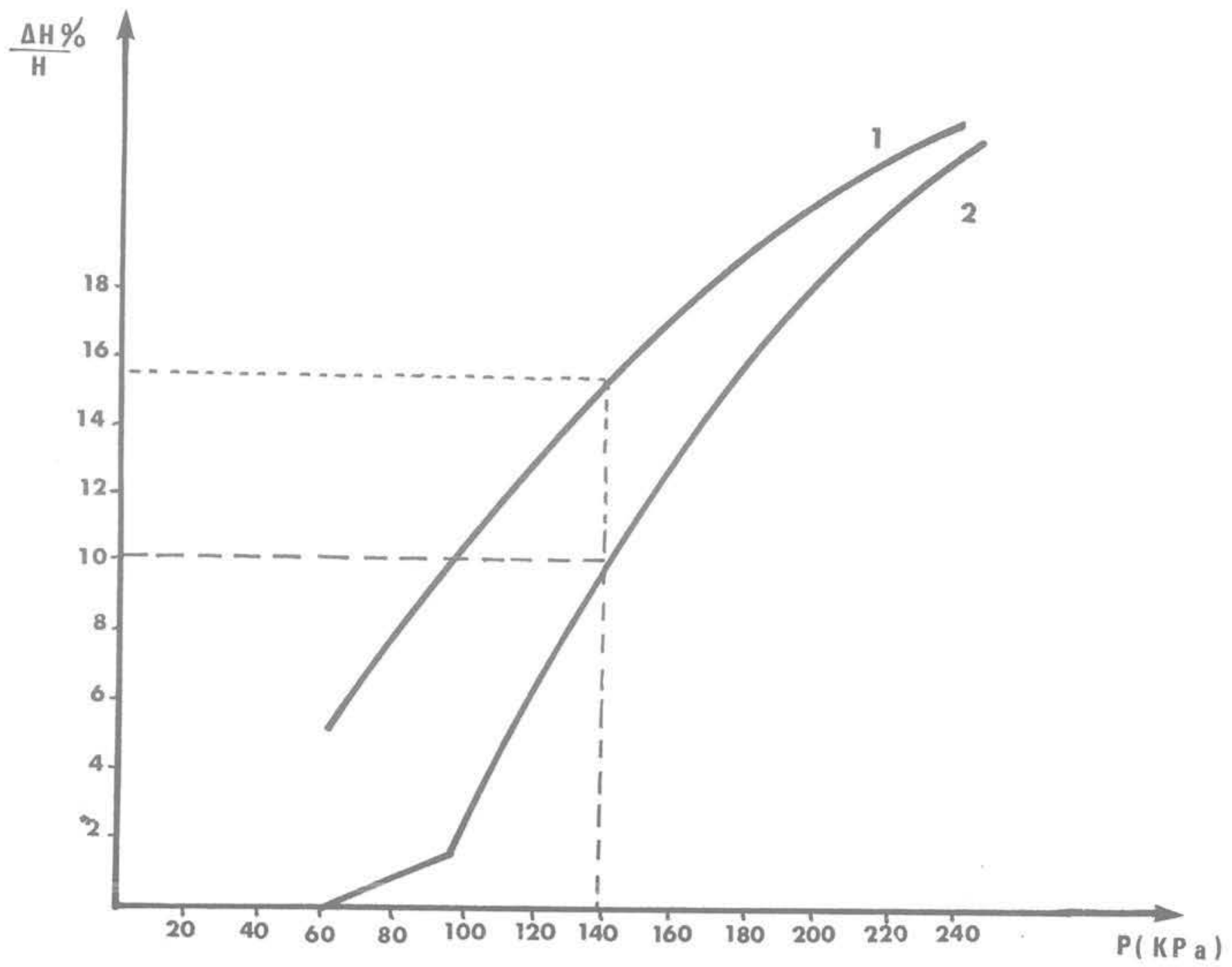




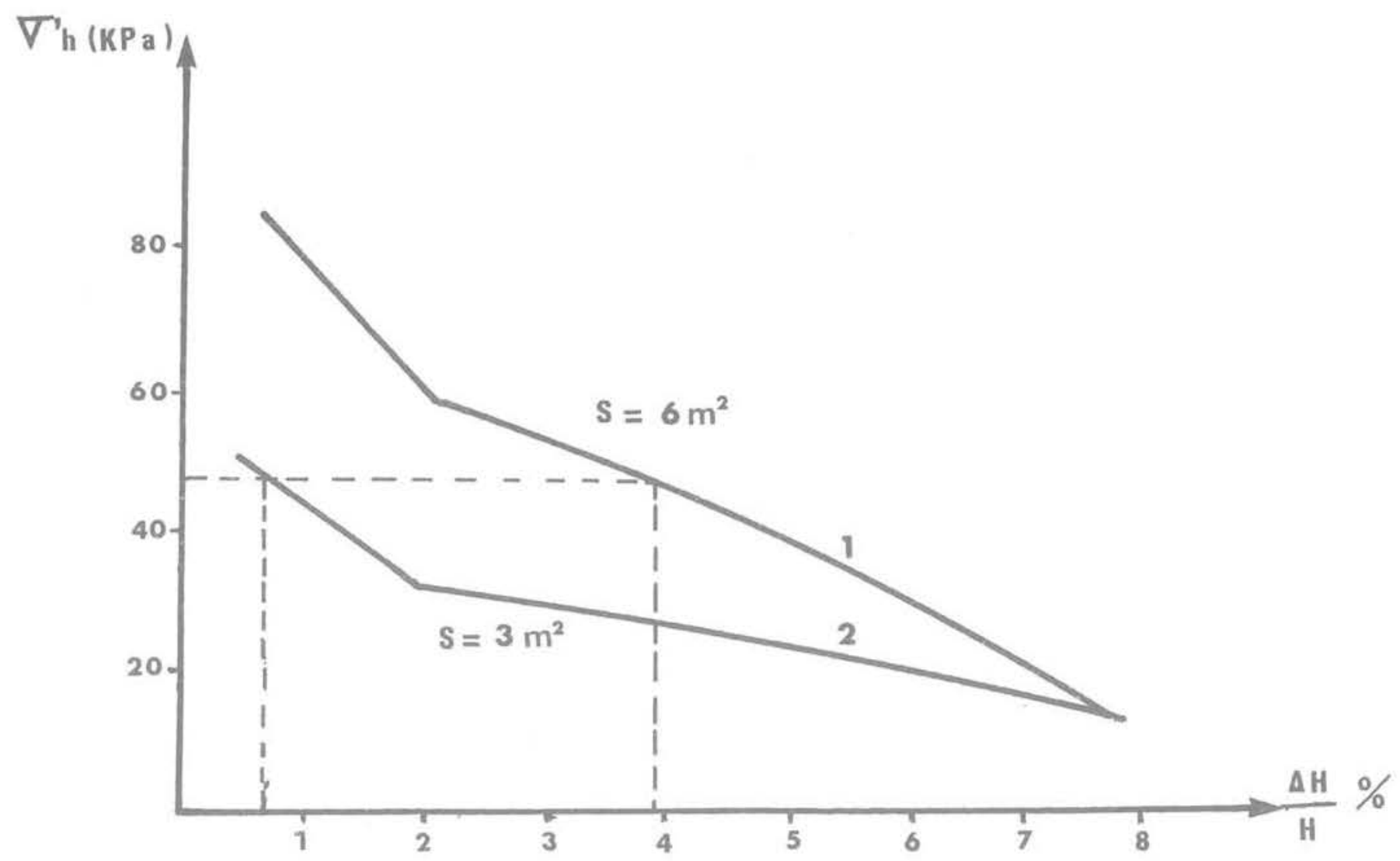

Fig. 18 Influence de la maille du traitement

\subsection{Remblai Rive Gauche}

Les mailles du traitement sont ici de $6 \mathrm{~m}^{2}$. Au cours de la première phase de construction, la charge $P$ était de $80 \mathrm{kPa}$.

\section{Contraintes horizontales}

La courbe 1 de la figure 18 donnant le tassement relatif de la couche de tourbe en fonction de la contrainte horizontale $\sigma_{h}^{\prime}$ montre que pour $4 \%$ de tassement, valeur réellement obtenue, la contrainte $\sigma_{h}^{\prime}$ est de $50 \mathrm{kPa}$, soit identique à celle mesurée rive droite et voisine de la valeur initiale.

\section{Influence de la maille du traitement}

La courbe 2 de la figure $n^{\circ} 18$ donne pour le même cas de charge $\mathrm{P}=80 \mathrm{kPa}$, le tassement calculé de cette couche de tourbe avec une maille de $3 \mathrm{~m}^{2}$.

II est possible de vérifier que :

- pour une maille de $3 \mathrm{~m}^{2}$, le tassement relatif serait inférieur à $1 \%$;

- pour une maille de $6 \mathrm{~m}^{2}$, le tassement serait de $4 \%$; - sans traitement, le tassement atteindrait $8 \%$.

\section{Conséquences}

\subsection{La reconnaissance}

Pour prévoir le comportement de colonnes ballastées, il est nécessaire de connaître :

- le poids spécifique $\gamma^{\prime}$ des sols rencontrés, afin de déterminer la pression des terres en profondeur.
- les caractéristiques de compressibilité du sol qui seront déterminées à l'œdomètre.

La reconnaissance devra donc comporter la réalisation de carottages avec prélèvements d'échantillons intacts.

\subsection{Contrainte horizontale dans le sol}

Les mesures qui ont été réalisées à Rouen montrent que la contrainte horizontale à prendre en compte dans les calculs prévisionnels serait celle qui a été mesurée entre les colonnes avant le chargement. Dans le cas présent, il s'avère qu'elle est égale à la contrainte effective verticale $\sigma_{v_{0}}^{\prime}$ calculée à partir des mesures de poids spécifiques sur carottage, lorsque le sol était déchargé de $1,50 \mathrm{~m}$ de remblai en vue d'encastrer le mur en terre armée. II semble donc que la fabrication des colonnes augmente la contrainte horizontale, mais ceci mériterait d'être vérifié au cours d'expérimentations similaires.

\subsection{Essai de chargement en vraie grandeur}

Un tel essai n'a d'intérêt que si la colonne est susceptible d'avoir un comportement élastique ou élastoplastique. La colonne agit alors à la manière d'un pieu. Cependant, la charge qu'elle est susceptible de porter de cette façon, est faible. Dans le cas d'un comportement plastique, les déformations sont celles du terrain naturel et sont définies par l'évolution des contraintes effectives au cours de la consolidation. Ce phénomène, de longue durée, ne peut être appréhendé avec un essai de chargement en vraie grandeur qui ne dure que quelques heures. 


\subsection{Cas des bâtiments rigides}

Pour ces constructions dont le tassement doit être faible, il convient de faire travailler la colonne en-deçà du déviateur de rupture, c'est-à-dire de rester dans le domaine des déformations élastiques ou élastoplastiques.

\section{Conclusion}

L'introduction de colonnes ballastées dans un sol compressibles est une technique intéressante qui a permis à Rouen d'éviter de prolonger le Pont Mathilde et ainsi de réaliser des économies importantes.

Cependant, le comportement de cet ensemble solcolonnes ainsi constitué, est complexe de telle sorte qu'il est difficile d'établir des prévisions. La méthode de calcul prévisionnel proposée à la suite de ces mesures et de leur interprétation semble donner des résultats satisfaisants.

Elle montre, en particulier, que le comportement du sol traité est lié aux contraintes effectives existant dans le sol et à leur évolution au cours du temps.

Mais le problème n'est pas totalement résolu, car il reste lié à des estimations telles que celles des caractéristiques géométriques et mécaniques des colonnes, et de l'état initial des contraintes horizontales dans le sol. De nombreuses expérimentations similaires devront être réalisées, si l'on veut affiner ces résultats et se donner des critères permettant de déterminer avec plus de précision les valeurs de ces estimations.

\section{Références Bibliographiques}

BLONDEAU F. - Les méthodes d'analyse de stabilité Bulletin de Liaison des Laboratoires des Ponts et chaussées - Stabilité des Talus - Numéro Spécial II Mars 1976 - pp. 56 à 62 .

BOURGES F. - Étude et Construction des remblais sur sols compressibles - Bulletin de Liaison des Laboratoires des Ponts et Chaussées - Remblais sur sols compressibles - Spécial T - Mai 1979 - pp. 10 à 23.

COMBARIEU O. - Effet d'accrochage et Méthode d'évaluation du frottement négatif - Bulletin de Liaison des Laboratoires des Ponts et Chaussées $n^{\circ} 71$ - 1974 pp. 93 à 107.

JARDIN J. - Traitements d'alluvions compressibles par pieux colonnes ballastés - Bulletin de Liaison des Laboratoires des Ponts et Chaussées $n^{\circ} 69$ - 1974 pp. 30 à 33 .

PILOT G. - La stabilité des remblais sur sols mous Bulletin de Liaison des Laboratoires des Ponts et Chaussées - Stabilité des Talus - Numéro Spécial III Décembre 1976 - pp. 83 à 106.

VAUTRAIN J. - Mur en terre armée sur colonnes ballastées - Géotechnical aspects of soft clays Proceeding of the International Symposium on soft clay - Bangkok, Thailland - Edited by RP Brennett and EW Brand - July 1977 - pp. 613 à 626. 
